A more general comment is that routine methods for diagnosing respiratory infection are unsatisfactory, as these papers show in different ways. Representative sputum samples are often difficult to obtain, sputum is invariably contaminated by commensals which can overgrow and obscure pathogens on culture, and transient residents of the mouth including Gram negative organisms and MRSA - may be cultured and potential pathogens may only be carrier organisms. Blood culture, as Jones et al show, is worthwhile when pneumonia is present.

Serological diagnosis in general requires a fourfold use in IgG titre which may not happen for some days and reagents for IgM antibody detection are often not readily available. Infections limited to respiratory surfaces may not stimulate antibodies, as in the case described by Pizzichini et al. PCR has yet to translate to routine diagnosis but, being very sensitive, it will detect carrier organisms. One route to better diagnosis is the greater use of techniques that directly sample the alveolar or bronchial secretions. Whether more detailed analysis of the cellular and chemical contents of sputum, as described by Pizzichini et al, ${ }^{2}$ will be useful requires wider experience.

In terms of treatment, does it matter that apparently rare pathogens, which might really be more common, are rarely diagnosed? Ery- thromycin and amoxycillin or a cephalosporin will cover many of them including mycoplasmas, legionellas, coxiella, chlamydias, $N$ meningitidis and some enterobacteriaceae. Initial treatment with these antibiotics remains appropriate provided adjustment is made for risk factors. If response is delayed beyond a few days, review and additional investigations are required.

1 Jones EM, Brown NM, Jarvey JE, Reeves DS, MacGowan AP. Three cases of meningococcal pneumonia. Thorax 1997;52:927-9.

2 Pizzichini MMM, Pizzichini E, Efthimiadis A, Clelland L, Mahony JB, Dolovich J, it al. Markers of inflammation in induced sputum in acute bronchitis caused by Chlamydic pneumomiae. Thorax 1997;52:929-31.

3 British Thoracic Society and the Public Health Laboratory Service. Community-acquired pneumonia in adults in British hospitals in 1982-1983: a survey of actiology, mortality, prognostic factors and outcome. 0 f Med (New Series 62) prognostic factors a

4 Kerttula Y, Leinonen M, Koskela M, Makela RPH. The etiology of pneumonia, application of bacterial serology and basic laboratory methods. F Infect 1987;14:21-30.

5 Kauppinen M, Saikku P. Pneumonia due to Chlamydia pneumoniae: prevalence, clinical features, diagnosis and treatment. Clin Infect Dis 1995;21(Suppl 3):5244-52.

6 Kuo CC, Jackson LA, Campbell LA, Grayston JT. Chlamydia pneumoniae (TWAR). Clin Microbiol Rev 1995;8:451-61.

7 Hyman CL, Roblin PM, Gaydos CA, Quinn TC, Schachter J, Hammerschlag MR. Prevalence of asymptomatic nasopharyngeal carriage of Chlamydia pnetumoniae in subjectively healthy adults: assessment by polymerase chain reactionenzyme immunoassay and culture. Clin Infect Dis 1995;20: $1174-8$

8 Thom DH, Grayston JT, Wang SP, Kuo CC, Altman J. Chlamydia pneumoniae strain TWAR, Mycoplasma pneumoniae and viral infection in acute respirators disease in a moniae and viral infection in acute resiol 1990;132:248-56.

9 Chirgwin K, Roblin PM, Gelling M, Hammerschlag MR, Schachter J. Infection with Chlamydia pnetumoniae in Brooklin. F Infect Dis 1991;163:757-61.

\section{Three cases of meningococcal} pneumonia

\author{
E M Jones, N M Brown, J E Harvey, \\ D S Reeves, A P MacGowan
}

\begin{abstract}
Three cases of pneumonia due to Neisseria meningitidis are described. In all three cases the organism was isolated only from blood cultures, but in the presence of good clinical and radiological evidence of pneumonia. The isolates belonged to three different serogroups: $B$ type $2 b, C$, and $Y$. The cases illustrate the fact that $N$ meningitidis can cause pneumonia and that culture of blood plays an important part in the diagnosis. Clinically there is nothing to differentiate meningococcal pneumonia from other causes of community acquired pneumonia. Predisposing factors include aspiration, immunosuppression, influenza, and adenovirus infections. When diagnosed, pneumonia due to $\boldsymbol{N}$ meningitidis should be notified and prophylaxis given as for meningitis or septicaemia. (Thorax 1997;52:927-929)
\end{abstract}

Keywords: Neisseria meningitidis, pneumonia, meningococcal disease.
Meningitis and septicaemia are well recognised features of infection with Neisseria meningitidis. Respiratory tract infections due to meningococci, though reported as long ago as 1906, ${ }^{12}$ remain rare. It is also not a cause of community acquired pneumonia specifically mentioned in the BTS survey. ${ }^{3}$ The three cases described here were all diagnosed by blood culture isolation of $N$ meningitidis associated with good clinical and radiological evidence of pneumonia.

\section{Case reports}

CASE 1

A 19 year old man presented with a sharp abdominal pain of sudden onset exacerbated by coughing following a night of heavy alcohol intake. On examination his temperature was $39^{\circ} \mathrm{C}$ and the pain had localised in the right side of his chest. The chest radiograph showed loss of definition of the right heart border with consolidation. No sputum was available for examination. Investigations revealed a haemoglobin of $14.6 \mathrm{~g} / \mathrm{dl}$ and white cell count of $13.8 \times 10 \%$. Blood cultures were taken and empirical treatment for aspiration pneumonia was started with cefuroxime $750 \mathrm{mg}$ intravenously eight hourly, clarithromycin $500 \mathrm{mg}$ orally 12-hourly, and metronidazole $500 \mathrm{mg}$ intravenously eight hourly. There was no clinical evidence of meningitis so a lumbar puncture was not performed. Two days after admission Gram negative coccobacilli were seen in the blood cultures which subsequently grew a group C $N$ meningitidis. Treatment was changed
Returned to authors 24 June 1996

Revised version receive
11 September 1996 Accepted for publication 2 October 1996 
to cefotaxime and the patient made a good recovery. The Department of Public Health Medicine was informed and prophylaxis was offered to close contacts. The patient also received two days of treatment with rifampicin $600 \mathrm{mg} \mathrm{12-}$ hourly to eradicate nasopharyngeal carriage of $N$ meningitidis. Atypical pneumonia serology was subsequently reported to be negative.

\section{CASE 2}

A 50 year old insulin dependent diabetic presented with sudden onset right sided pleuritic chest pain, haemoptysis, and dyspnoea. There was no history of headache or neck stiffiness. On examination he was tachypnoeic and in pain. Oxygen saturation on air was $82 \%$ and his temperature was $38^{\circ} \mathrm{C}$. Examination of the chest was consistent with the chest radiographic appearance of right basal consolidation. Investigations revealed a haemoglobin of $12.9 \mathrm{~g} /$ $\mathrm{dl}$ and a white cell count of $16.7 \times 10^{\circ} / 1$. Sputum was produced for analysis but yielded no growth. Treatment was started with cefuroxime $1.5 \mathrm{~g}$ intravenously eight hourly and erythromycin $500 \mathrm{mg}$ intravenously six hourly. Blood cultures grew a group B (type 2b) $N$ meningitidis. The Department of Public Health Medicine was notified but prophylaxis was not offered because of the time that had elapsed before the diagnosis was made. The patient made a full recovery. The atypical pneumonia serology was subsequently reported to be negative.

\section{CASE 3}

An 88 year old woman was admitted to hospital from respite care with a history of right sided lower chest pain and dyspnoea. The chest radiograph showed a right basal consolidation. She was not producing any sputum. There was no history of headache or neck stifiness. Other investigations revealed an arterial oxygen saturation of $88 \%$ on air, haemoglobin of 12.4 $\mathrm{g} / \mathrm{dl}$, and a white cell count of $27.8 \times 10^{\%} / 1$, predominantly neutrophils. Empirical treatment was started with cefuroxime $750 \mathrm{mg}$ intravenously eight hourly and initially she improved. Blood cultures taken on admission grew a group Y $N$ meningitidis. The Department of Public Health Medicine was notified and prophylaxis was given to close family members and to contacts in the respite care home. Treatment with cefuroxime was continued due to the initial good response. However, she later deteriorated and, despite several changes of antibiotic therapy, she died of her pneumonia 11 days after admission.

\section{Discussion}

Primary meningococcal pneumonia has been defined as a pneumonia caused by $N$ meningitidis without evidence of concomitant meningeal involvement or the WaterhouseFriderichsen syndrome. ${ }^{\prime}$ Clinically there is nothing to differentiate it from other causes of pneumonia, as shown by our cases.

The identification of three cases of meningococcal pneumonia between June and December 1994 prompted us to review the literature. It was a surprise to find that cases had been reported as far back as $1906 .^{12}$ It was not, however, a cause of community acquired pneumonia included in the BTS survey on aetiology, prognostic factors, mortality and outcome conducted between 1982 and 1983 . $^{3}$

Early cases of meningococcal pneumonia were associated with outbreaks of influenza such as the epidemic of 1918-19. ${ }^{45}$ Since then meningococcal pneumonia has rarely been described and its association with influenza has been less marked. Other predisposing factors are aspiration ${ }^{67}$ and immunosuppression. ${ }^{\circ}$ Our first case probably developed his pneumonia after aspiration. There was, however, no evidence of immunosuppression in either of the other cases although case 2 was diabetic and case 3 was a frail and elderly patient.

$N$ meningitidis of serogroup $\mathrm{Y}$ is particularly associated with meningococcal pneumonia. ${ }^{10}$ Since $197367 \%$ of meningococcal infections reported as pneumonias or respiratory tract infections have been due to $N$ meningitidis serogroup Y. ${ }^{2}$ However, this organism was only isolated from one of our cases.

The diagnosis of meningococcal pneumonia in the three cases described was made on the basis of a clinical picture followed by the isolation of $N$ meningitidis from blood cultures. Sputum was only obtained from one of our patients and yielded no growth. $N$ meningitidis is, however, a normal commensal of the upper respiratory tract and therefore unless it is isolated in pure or predominant growth it would probably be discarded. It is also therefore not selectively looked for in sputum specimens. For this reason blood cultures become an important investigation when a pneumonia presents.

All three of our cases were notified to the Department of Public Health Medicine and prophylaxis was offered to the contacts of two of the three patients. Evidence of transmission of $N$ meningitidis from a case of pneumonia has appeared in the literature ${ }^{7}$ and it may be that offering prophylaxis to contacts of this form of meningococcal disease is more important than for any other, as a coughing and spluttering patient may be more efficient at transmitting infection than someone with meningitis.

We conclude that $N$ memingitidis can cause respiratory tract infections but there are no defining clinical features. Blood cultures are an important investigation in community acquired pneumonia. $N$ meningitidis of serogroup $\mathrm{Y}$ is particularly associated with respiratory tract infections, although other serogroups can and do cause pneumonia. The standard empirical treatment for community acquired pneumonias as recommended by the $\mathrm{BTS}^{11}$ is appropriate for the treatment of pneumonia due to $\mathrm{N}$ meningitidis. A change to penicillin alone may also be considered after the diagnosis has been made and sensitivities are available. Meningococcal septicaemia is a notifiable disease and prophylaxis should be offered to close contacts of an individual with meningococcal pneumonia.

The authors would like to thank Dr A C Buck, Consultant Microbiologist, Princess Margaret Hospital, Swindon for providing details of the third case and Dr S Hughes, Consultan Physician at Southmead Hospital, Bristol for permission to report case 2. Thanks are also due to the Manchester PHLS meningococcal reference laboratory for typing the isolates. 
1 Darnell JC, Brandt MJ. Primary meningococcal pneumonia: a report of three cases. I Indiana St Mod Assoc 1981; $794-8$

2 Putsch RW, Hamilton JD, Wolinsky E. Neisseria meningitidis, a respiratory pathogen? I Infot Dis 1970;121:48-54.

3 Subcommitte of the Research Committe of the British Thoracic Society and the Public Health Laboratory Service. Community-acquired pneumonia in adults in British hospitals in 1982-1983: a survey of actiology, mortality; prognostic factors and outcome. $Q$ f Med (Niv Series) 1987;62:195-220

4 Fletcher W. Meningococcus bronchopneumonia in influenza. Lancet 1919:104.

5 Holm ML, Davison WC. Meningococcus pneumonia. 1 The occurrence of post-influenzal pneumonia in which the Diplococcus intracellularis meningitidis was isolated from the observations at Camp Coetquidon, AEF, France. Bull the observations at Camp Coetcu
Hopks Hosp 1919;30:324-9.
6 Barnes RV, Dopp AC, Gelberg HJ, Silva J. Nicisseria meningitidis: a cause of nosocomial pneumonia. Am Rev Respir Dis 1975;111:229-31.

7 Rose HD, Lenz IE, Sheth NK. Meningococcal pneumonia a source of nosocomial infection. Arh Intem Med 1981; $141: 575-7$

8 Winter RA, Helfgott D, Storey-Johnson C, Murray HW. Human immunodeficiency virus infection and bacteremic meningococcal pneumonia. F Infect Dis 1991;163:1390.

9 Hanson MF, Lawson A. Isolation of a group $Y$ meningococcus from a patient with pneumonia. f Infect 1985; 10:76-9.

10 Yee NM, Kat\% M, Neu HC. Meningitis, pneumonitis and arthritis caused by Neisseria meningitidis group Y. $\mathcal{F} A M A$ arthritis caused by

11 British Thoracic Society, London. Guidelines for the management of community-acquired pneumonia in adults admitted to hospital. Br f Hosp Med 1993;49:346-50.

\section{Markers of inflammation in induced sputum in acute bronchitis caused by Chlamydia pneumoniae}

\author{
M M M Pizzichini, E Pizzichini, \\ A Efthimiadis, L Clelland, J B Mahony, \\ J Dolovich, F E Hargreave
}

\begin{abstract}
Little is known of the inflammatory characteristics of acute infections of the respiratory tract caused by virus and unusual bacteria such as Chlamydia pneumoniae. A case is reported in whom inflammatory indices in sputum were used to investigate, for the first time, the airway inflammation during an episode of acute bronchitis caused by $C$ pneumoniae. The patient presented with a dry cough of five days duration. $C$ pneumoniae was identified by polymerase chain reaction (PCR) in a nasopharyngeal swab collected on day 5 . Virological studies were negative. Clinical and inflammatory indices in induced sputum were measured on days 6,8 , and 11 . The cough cleared spontaneously by day 11. Forced expiratory volume in one second was normal throughout. Sputum findings identified intense airway inflammation characterised by increased total cell and lymphocyte counts followed by an increase in neutrophils and a decrease in the $\mathrm{CD} 4 / \mathrm{CD} 8$ ratio, activation of CD8 lymphocytes, and exudation as indicated by an increase in fluid phase fibrinogen. These observations suggest that sputum might be useful to monitor an inflammatory/immune response of the airway in acute infections.

(Thorax 1997;52:929-931)
\end{abstract}

Keywords: Chlamydia pneumoniae, sputum, flow cytometry.

The study of airway inflammation in acute respiratory infections caused by viruses and unusual bacteria such as Chlamydia pneumoniae and Mycoplasma pneumoniae has been difficult because previous methods of examination have been invasive. Consequently, little is known about the magnitude of lower airway involvement and the kinetics of the airway inflammatory response during the acute phase or recovery and the effects of treatment. Current information in humans is limited to rhinovirus experimental colds examined by bronchial biopsy or bronchoalveolar lavage. ${ }^{2}$ Improvement of knowledge in this area is relevant for several reasons: (1) acute infections of the respiratory tract are the most frequent cause of illness; (2) they are an important cause of disability and days lost from work or school; ${ }^{3}$ and (3) they are frequently associated with asthma exacerbations."

During the last decade there has been a resurgence of interest in sputum examination to measure indices of airway inflammation directly, and indices of airway inflammation can now be measured relatively non-invasively and reliably in induced sputum. ${ }^{j-i}$ In addition, the examination of sputum separated from saliva has been shown to have longitudinal responsiveness $^{\text {s9 }}$ and can therefore be repeatedly applied to follow the course of the disease and the effects of treatment.

$C$ pneumoniae is an obligate intracellular Gram negative bacteria, primarily but not exclusively a respiratory pathogen, to which $30-$ $50 \%$ of adults worldwide are seropositive. ${ }^{10}$ It has been reported as a cause of pneumonia, bronchitis, sinusitis and pharyngitis ${ }^{11.1 .4}$ and may cause, more frequently than is recognised, exacerbations of asthma. ${ }^{15}$ To date, no information is available on the airway inflammatory response to $C$ pnemmoniae infection. We have therefore used induced sputum to follow longitudinal changes in the airway inflammatory response of a healthy subject during an episode of acute bronchitis caused by $C$ pneumoniae.

\section{Case report}

A previously healthy woman aged 42 years who was a non-smoker and non-atopic presented with a five day history of a watery nose, sore throat, myalgias, and bothersome dry cough disturbing sleep, but no fever. Physical examination was normal except for a red throat. The forced expiratory volume in one second $\left(\mathrm{FEV}_{1}\right)$ was 2.91 (100\% predicted) and slow vital capacity was 3.11 ( $92 \%$ predicted). She was reviewed on days 6,8 , and 11 . The symptoms disappeared by day 11 without any treatment. $\mathrm{FEV}_{1}$ was unchanged. A methacholine 